\title{
An AMC Backed Folded Dipole Slot Antenna Based on CMOS Process
}

\author{
Guo Qing Luo, Lin Qi Wu, and Xiao Hong Zhang \\ The Key Laboratory of RF Circuits and System of Ministry of Education, Hangzhou Dianzi University, Hangzhou 310018, China \\ Correspondence should be addressed to Guo Qing Luo; gqluo@emfield.org
}

Received 2 September 2013; Accepted 10 October 2013

Academic Editor: Bing Liu

Copyright (C) 2013 Guo Qing Luo et al. This is an open access article distributed under the Creative Commons Attribution License, which permits unrestricted use, distribution, and reproduction in any medium, provided the original work is properly cited.

A fold dipole slot antenna backed by artificial magnetic conductor (AMC) structure based on a standard 0.18 um CMOS process on chip application is firstly proposed in this paper. Conventional silicon antenna on chip (AoC) suffers from low radiation performance because the most electromagnetic energy is restricted in silicon substrate as surface wave for its high dielectric permittivity. The energy is dissipated as thermal for low resistivity of silicon substrate. AMC constructed by a periodic $6 * 6$ square patch array is adopted as background to improve radiation performance of the proposed folded dipole slot AoC. Gain of the proposed AMC backed AoC is improved about $3.5 \mathrm{~dB}$ compared with that of the same AoC without AMC background.

\section{Introduction}

Millimeter wave is a kind of electromagnetic wave whose frequency spectrum is from $30 \mathrm{GHz}$ to $300 \mathrm{GHz}$ and its corresponding wavelength varies from 10 to $1 \mathrm{~mm}$. People pay more attention to the millimeter wave band according to the lack of available spectrum in radio frequency and microwave band. Different kinds of millimeter wave devices and systems have been exploited. Antenna, as an indispensable device of transmitting and/or receiving energy in wireless system, will directly affect performance of the whole wireless terminal system. With the development of wireless technology, people put forward higher requirements for the performance of antenna, which are specifically in the following areas: size reduction, broad bandwidth, multiband operation, and so forth. $140 \mathrm{GHz}$ band is at the overlap of millimeter wave and terahertz wave (its frequency is 0.1 $10 \mathrm{THz}$, wavelength is $3 \mathrm{~mm} \sim 30 \mathrm{um}$ ), whose development mainly depends on the technology of electronics. Terahertz is a band which is not completely recognized and explored by human beings. However, with the continuous development of stable terahertz sources during the past decade, recognization of terahertz communication increases gradually. Compared with microwave communication, the capacity of terahertz communication has a great promotion, which can support a high communication rate up to $10 \mathrm{~GB} / \mathrm{s}$. Terahertz wave has better directivity, higher resolution and security, greater anti-interference ability, and smaller wavelength. Compared with optical communication, terahertz communication has a higher energy efficiency and a better penetrability.

For millimeter wave integrated circuit, CMOS technology is an excellent technical platform because it has the virtues of low cost, light weight, and so on. Recently antenna integration with RF front end circuit in a single wafer has been extensively investigated. Once the antenna integration realized successfully, the whole size of RF front end system will be very compact. Meanwhile, the interconnection loss between antenna and RF front end circuit will be greatly reduced. Therefore, the conception of antenna on chip (AoC) has been proposed. From the published literature it can be found that the forms of presented $\mathrm{AoC}$ are various [1-4]. A common problem of AoC is that its radiation efficiency is very low for the high dielectric constant and low resistivity of silicon substrate. Many ways can be used to improve AoC performance [5-10], such as removing silicon substrate under radiating element by micromachining, using ion implanting technology to increase silicon substrate resistivity. However, these improved methods need postprocessing, which will improve the cost. Therefore, high radiation efficiency antennas manufactured by the standard silicon process are the most required. 


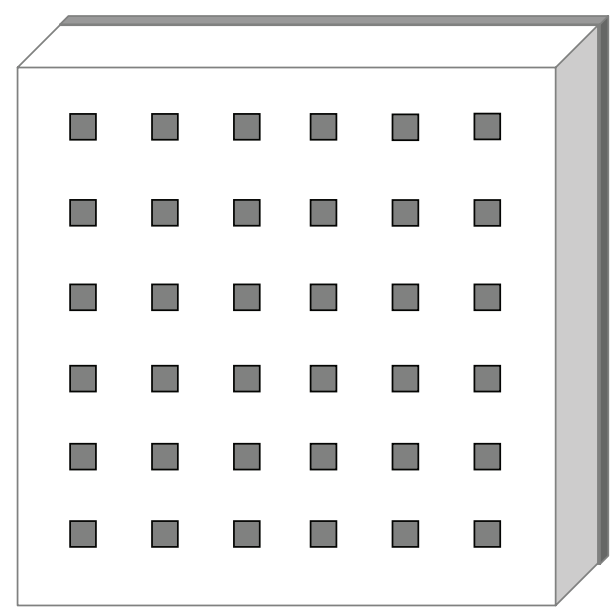

FIGURE 1: The proposed AMC structure.

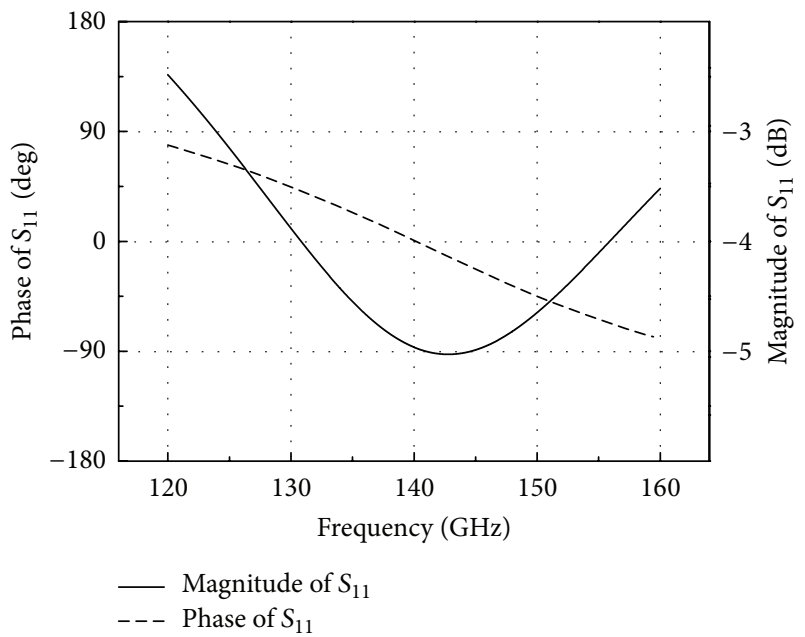

Figure 2: Phase and magnitude of reflection coefficient of the proposed AMC.

\section{AMC}

Artificial magnetic conductor (AMC), as a kind of periodic structure, has the same total reflection characteristic as that of perfect electric conductor (PEC) when they are excited by incident waves. A fundamental difference between these two materials is that $\mathrm{AMC}$ introduces a zero degree reflection phase shift to an incident wave but PEC introduces a 180degree reflection phase shift to an incident wave. This special characteristic makes AMC suitable for application in low profile antenna design, in which efficiency of antenna will be improved if its radiating element is set more close to the AMC background.

Recently AMCs have been extensively used in low profile antennas and integrated antennas designs. In this paper, an AMC backed folded dipole slot antenna is proposed and investigated. The proposed AMC structure is plotted in Figure 1. It consists of three layers. The bottom layer is a full

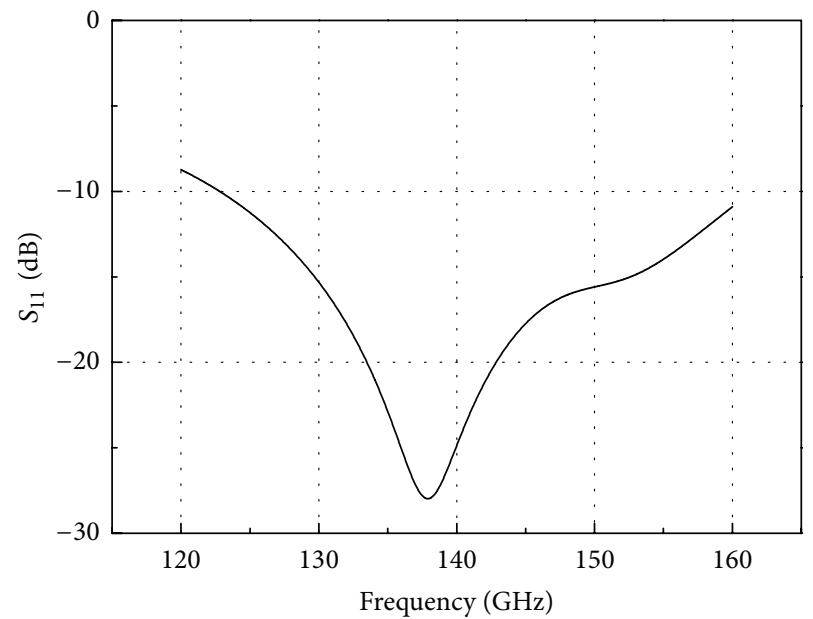

(a)

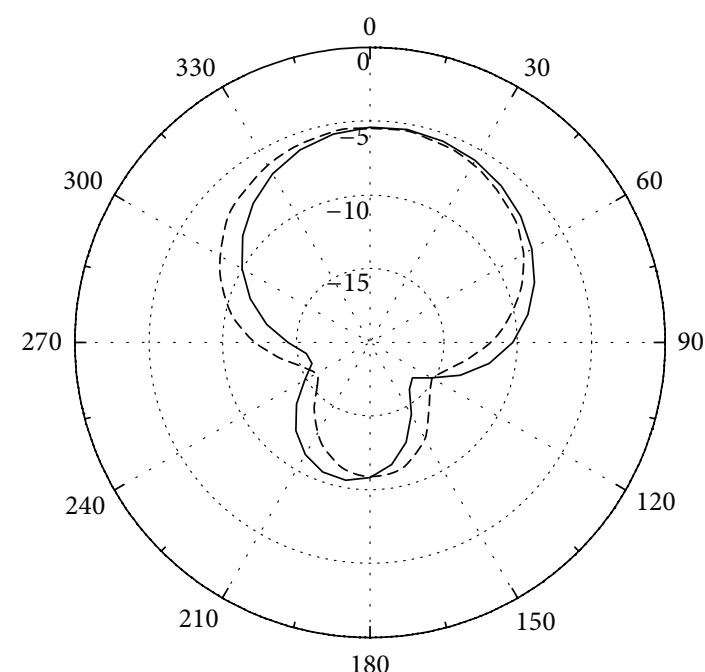

$\begin{array}{ll}\text { - E-plane } \\ --- & \text { H-plane }\end{array}$

(b)

FIgURE 3: (a) $S_{11}$ of a folded dipole slot antenna without AMC. (b) Radiation pattern of a folded dipole slot antenna without AMC.
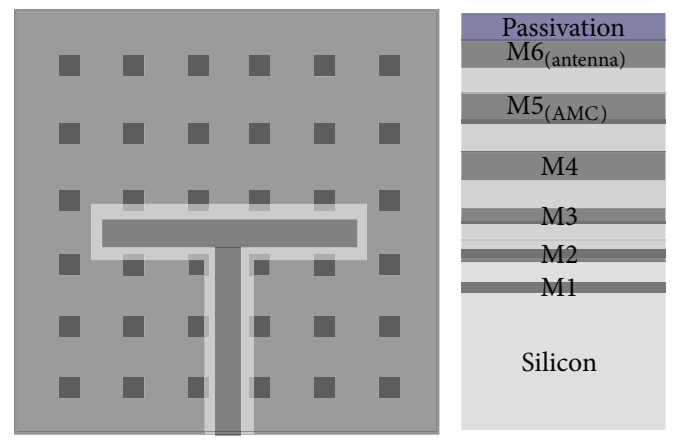

Figure 4: A folded dipole slot antenna backed by AMC. 


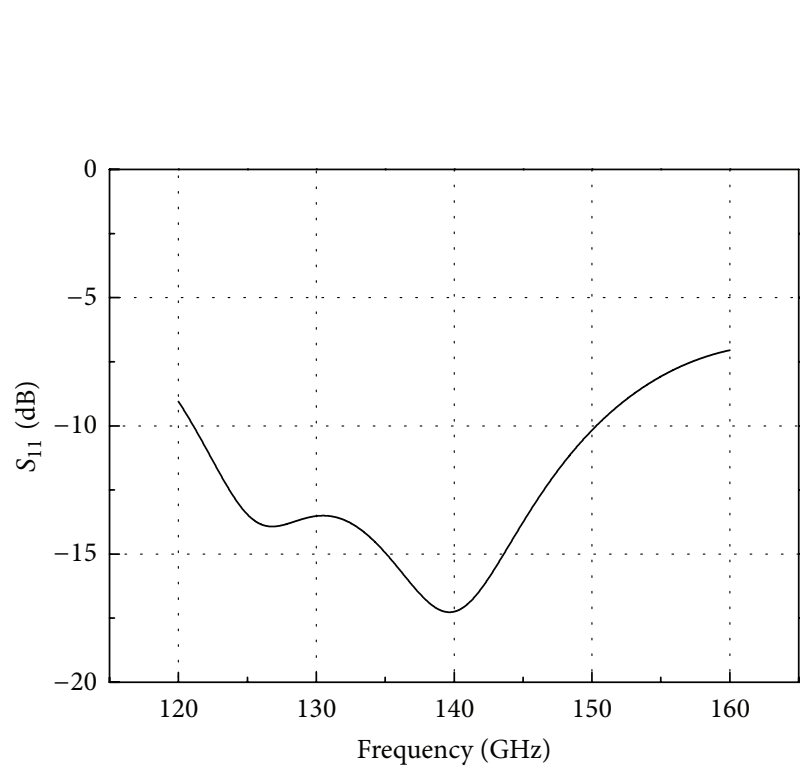

(a)

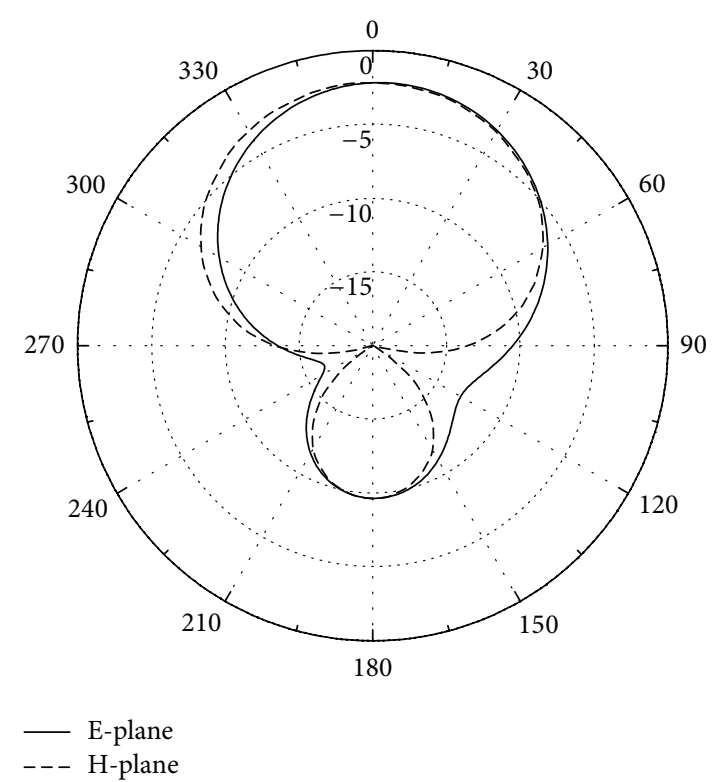

(b)

FIGURE 5: (a) $S_{11}$ of a folded dipole slot antenna backed by AMC. (b) Radiation pattern of a folded dipole slot antenna backed by AMC.

metallic plane. The intermediate layer is a dielectric substrate layer. The top layer is a periodic square metallic patch array.

Frequency response of the proposed AMC is shown in Figure 2, in which it is calculated from that the AMC excited by a normally incident plane wave. From the phase and magnitude curves of the AMC reflection coefficient it can be found that the proposed AMC is operating at $140 \mathrm{GHz}$ and its fractional operating bandwidth is about 25\% (from $125 \mathrm{GHz}$ to $160 \mathrm{GHz}$ when phase varied from $90^{\circ}$ to $-90^{\circ}$ ). These results indicate that the proposed AMC can be used in low profile AoC designs.

\section{AoC}

A standard 0.18 um CMOS process is employed in AoC design. The standard 0.18 um CMOS structure consisted of six metal layers (copper or aluminum) and one polysilicon layer. There is silicon dioxide or its derivatives in metal layers with a relative dielectric constant of 4 . There are two passivation layers above the top metal layer M6, which are silicon dioxide derivatives and silicon nitride. These two passivation layers are applied to prevent the whole circuit from oxidation and thus its performance deterioration. There is a doping silicon substrate about $290 \mathrm{~nm}$ thick below the polysilicon layer, whose relative dielectric constant is 11.9 and resistivity is about $10 \Omega \cdot \mathrm{cm}$. In particular, the conductivity of metal layers from M6 to M1 will gradually fade out. In order to maintain antenna performance the top metal layer M6 is usually used to fabricate the antenna.

In this paper AoC is a folded dipole slot, which is realized on the top metal layer (M6). The folded dipole slot antenna is fed by a $50 \mathrm{ohm}$ coplanar waveguide. The proposed AoC without AMC background is firstly studied. Its frequency response and radiation performance are shown in Figure 3.
From these figures it can be found that the proposed AoC operates at $140 \mathrm{GHz}$ with a matched impedance bandwidth more than $35 \mathrm{GHz}$. A peak gain of $-5.5 \mathrm{~dB}$ and a back lobe level of $-11 \mathrm{~dB}$ have been achieved. Radiation efficiency of the proposed AoC is very low because of the high loss characteristic of the silicon substrate. Most electromagnetic energy is restricted in the silicon substrate as surface wave and it is dissipated as thermal for the low resistivity of the silicon substrate.

In order to improve radiation efficiency of the proposed AoC, AMC is adopted as background inserted between the folded dipole slot antenna and the silicon substrate. Electromagnetic wave radiating from the antenna will be prevented propagating into the silicon substrate by the AMC background plane. Furthermore, this electromagnetic wave will be fully reflected by the AMC background without phase shift. It will be superposed with the positive radiating wave in phase and then a gain enhanced radiation is achieved.

Figure 4 shows a folded dipole slot antenna with an AMC background. The proposed AMC is constructed by a periodic $6 * 6$ square patch array at the middle metallic layer M5. Its frequency response and radiation performance are shown in Figure 5. From these figures it can be found that the proposed AoC backed by AMC has a matched impedance bandwidth more than $29 \mathrm{GHz}$. A peak gain of $-2 \mathrm{~dB}$ and a back lobe level of $-10 \mathrm{~dB}$ have been achieved. Compared with AoC without AMC presented in Section 3, the peak gain of AoC with AMC has been improved about $3.5 \mathrm{~dB}$. Its radiation efficiency has been enhanced more than $100 \%$.

\section{Conclusions}

In this paper, an AMC backed AoC constructed by a standard 0.18 um CMOS process is presented. The AoC is a folded 
dipole slot antenna located at the top metal layer M6 and the AMC is constructed of a periodic $6 * 6$ square patch array at the middle metal layer M5. By using AMC as background plane between the folded dipole slot antenna and the silicon substrate, peak gain of the proposed AoC has been improved about $3.5 \mathrm{~dB}$ and its radiation efficiency has been enhanced more than $100 \%$.

\section{Acknowledgments}

This work was supported in part by the National Basic Research Program of China under Contract 2010CB327403, the National Science and Technology Major Project of China under Contract 2010ZX03007-001-01, the NSFC under Contract 61372020, the ZJNSF under Contract R1110003, and the FANEDD under Contract 201045.

\section{References}

[1] K. T. Chan, A. Chin, Y. D. Lin et al., "Integrated antennas on Si with over $100 \mathrm{GHz}$ performance, fabricated using an optimized proton implantation process," IEEE Microwave and Wireless Components Letters, vol. 13, no. 11, pp. 487-489, 2003.

[2] C.-C. Lin, S.-S. Hsu, C.-Y. Hsu, and H.-R. Chuang, "A 60-GHz millimeter-wave CMOS RFIC-on-chip triangular monopole antenna for WPAN applications," in Proceedings of the IEEE Antennas and Propagation Society International Symposium, pp. 2522-2525, June 2007.

[3] A. Babakhani, X. Guan, A. Komijani, A. Natarajan, and A. Hajimiri, "A 77-GHz phased-array transceiver with on-chip antennas in silicon: receiver and antennas," IEEE Journal of Solid-State Circuits, vol. 41, no. 12, pp. 2795-2805, 2006.

[4] C.-S. Wang, J.-W. Huang, S.-H. Wen, S.-H. Yeh, and C.-K. Wang, "A CMOS RF front-end with on-chip antenna for Vband broadband wireless communications," in Proceedings of the 33rd European Solid State Circuits Conference, pp. 143-146, September 2007.

[5] S. Pan and F. Capolino, "Design of a CMOS on-chip slot antenna with extremely flat cavity at $140 \mathrm{GHz}$," IEEE Antennas and Wireless Propagation Letters, vol. 10, pp. 827-830, 2011.

[6] S. Pan, D. Wang, and F. Capolino, "Novel high efficiency CMOS on-chip antenna structures at millimeter waves," in Proceedings of the IEEE International Symposium on Antennas and Propagation (APSURSI '11), pp. 907-910, July 2011.

[7] E. Öjefors, H. Kratz, K. Grenier, R. Plana, and A. Rydberg, "Micromachined loop antennas on low resistivity silicon substrates," IEEE Transactions on Antennas and Propagation, vol. 54, no. 12, pp. 3593-3601, 2006.

[8] B. Biglarbegian, M. R. Nezhad-Ahmadi, C. Hoggat, S. Hose, M. Fakharzadeh, and S. Safavi-Naeini, "A $60 \mathrm{GHz}$ on-chip slot antenna in silicon integrated passive device technology," in Proceedings of the IEEE Antennas and Propagation Society International Symposium (APSURSI '10), pp. 1-4, July 2010.

[9] A. Fonte, S. Saponara, G. Pinto, and B. Neri, "Feasibility study and on-chip antenna for fully integrated $\mu$ RFID tag at $60 \mathrm{GHz}$ in $65 \mathrm{~nm}$ CMOS SOI," in Proceedings of the IEEE International Conference on RFID-Technologies and Applications (RFID-TA '11), pp. 457-462, September 2011.
[10] S. Pan, D. Wang, C. Guclu, and F. Capolino, "High impedance layer for CMOS on-chip antenna at millimeter waves," in Proceedings of the IEEE International Symposium on Antennas and Propagation (APSURSI '11), pp. 903-906, July 2011. 

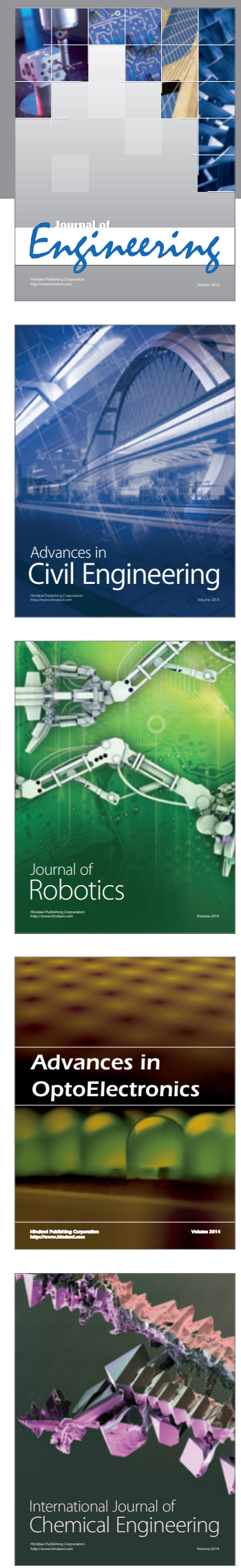

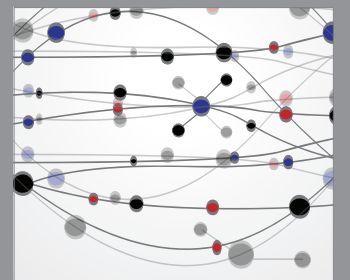

The Scientific World Journal
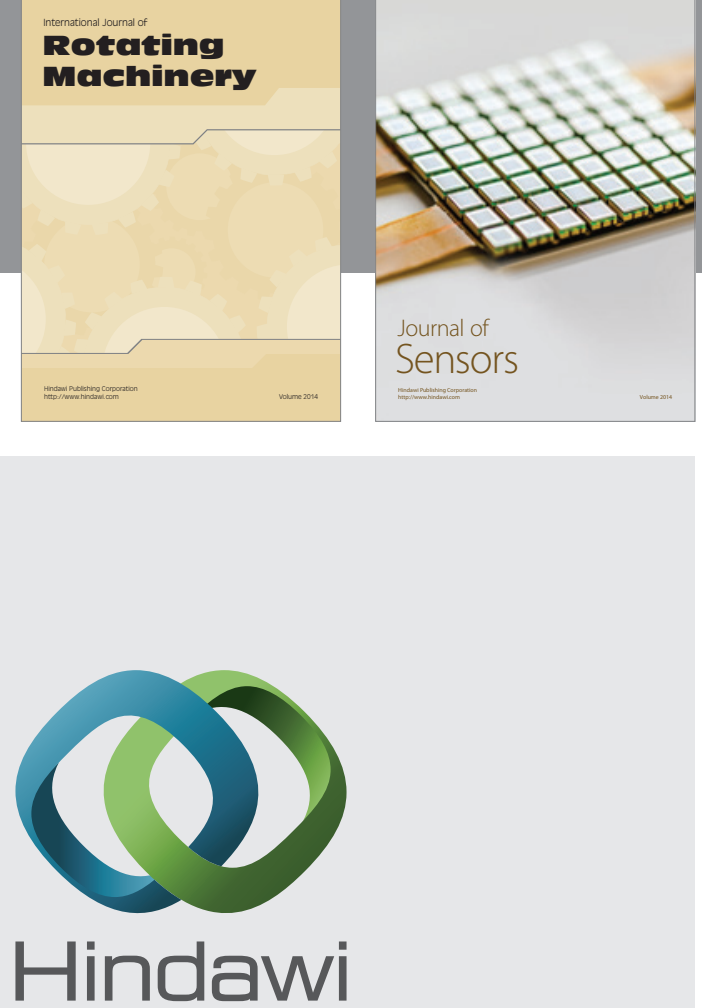

Submit your manuscripts at http://www.hindawi.com
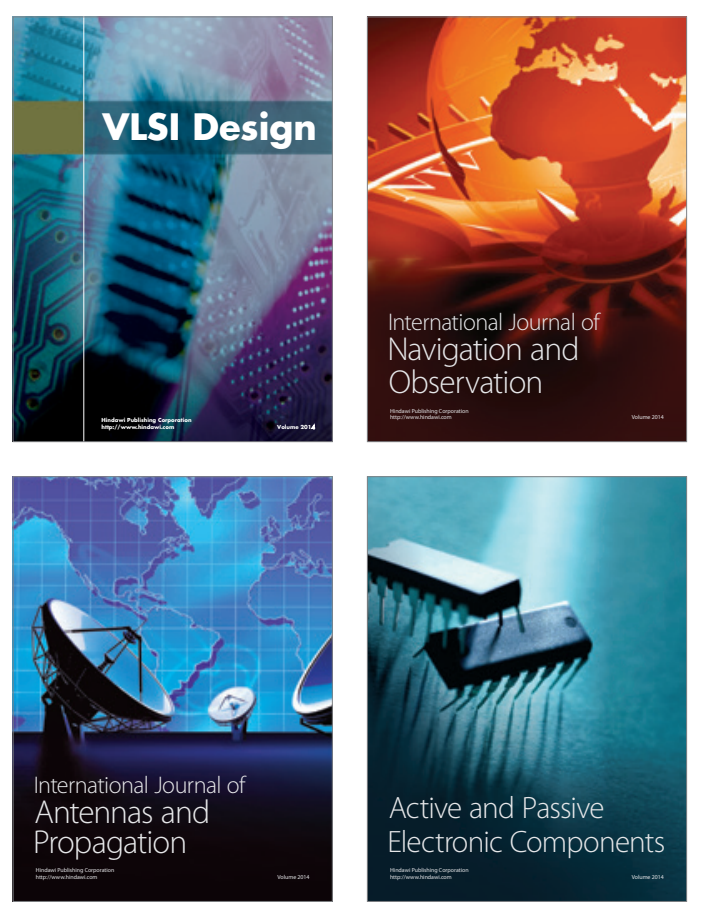
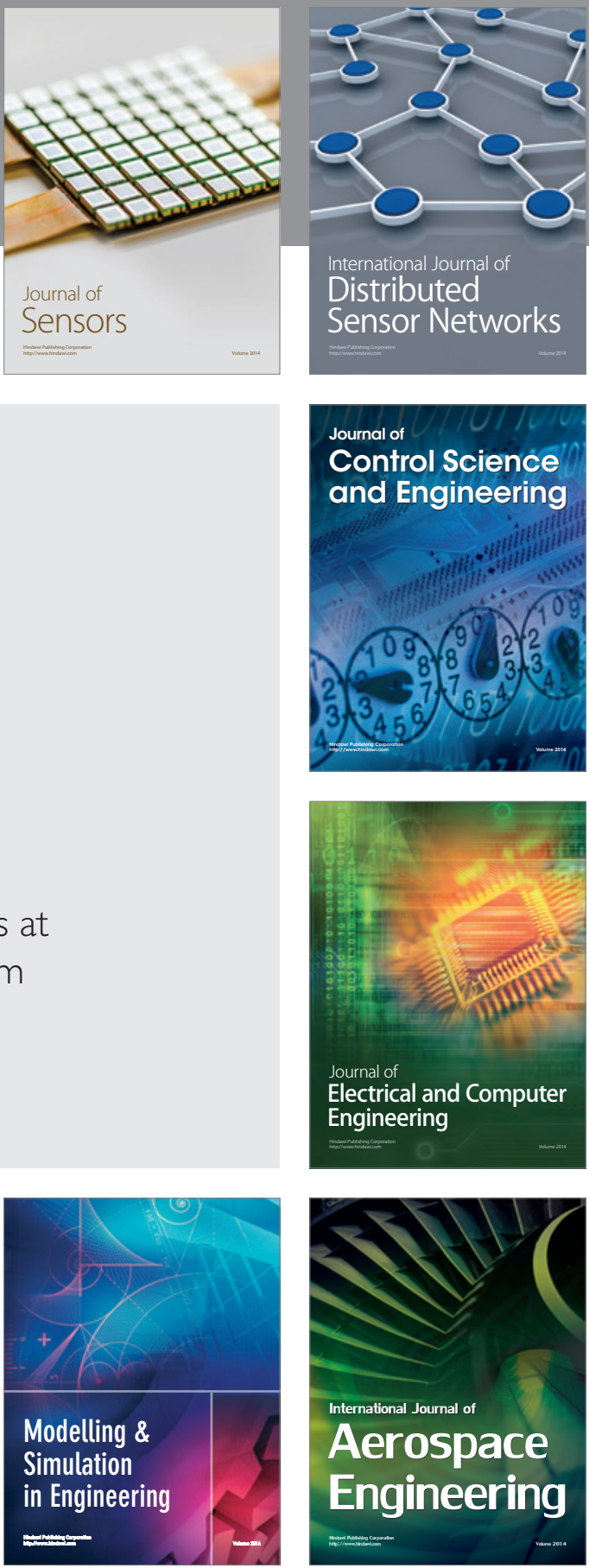

Journal of

Control Science

and Engineering
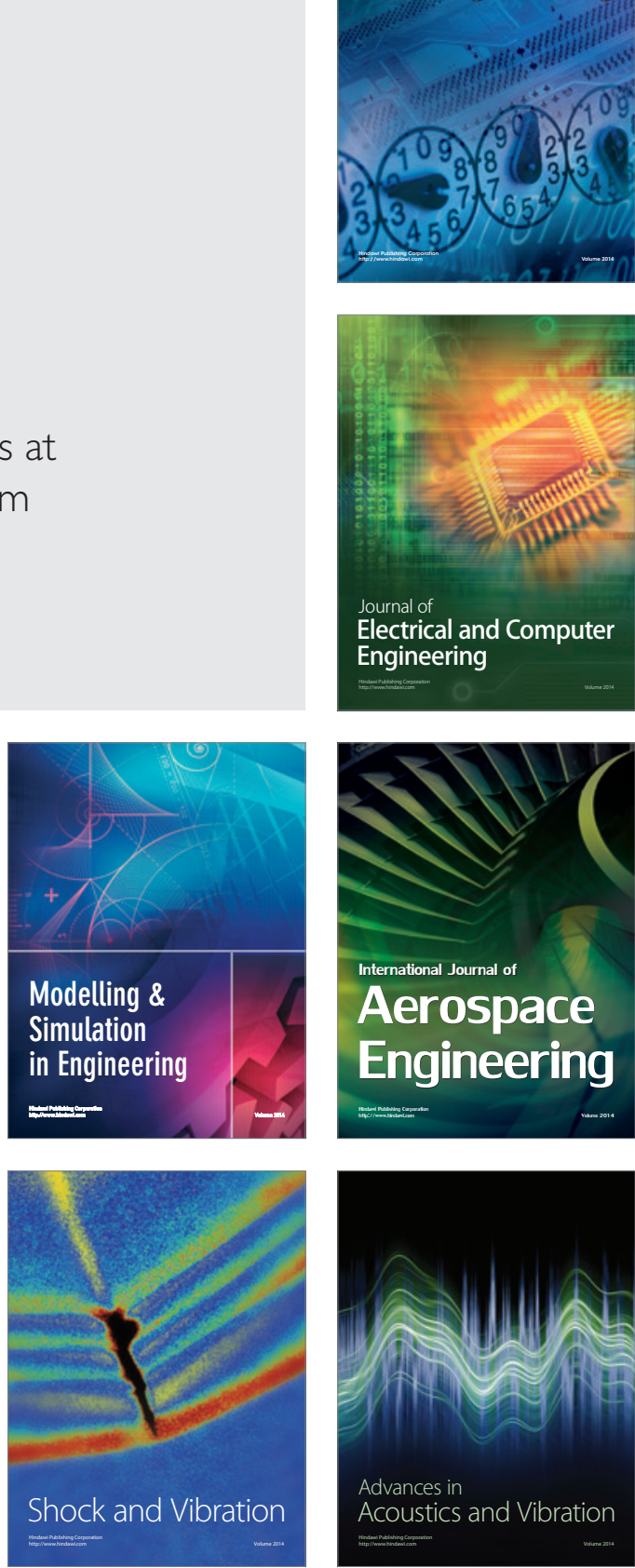